\title{
A Study to Investigate the Effect of 'Frontal Lift' Osteopathic Manipulative Technique (OMT) in Patients with Chronic Sinusitis
}

\author{
David Lintonbon* \\ British College of Osteopathic Medicine, UK \\ *Corresponding author: David Lintonbon, British College of Osteopathic Medicine, UK. \\ To Cite This Article: David Lintonbon. A Study to Investigate the Effect of 'Frontal Lift' Osteopathic Manipulative Technique (OMT) in Patients \\ with Chronic Sinusitis. Am J Biomed Sci \& Res. 2019 - 3(3). AJBSR.MS.ID.000665. DOI: 10.34297/AJBSR.2019.03.000665
}

Received: May 08, 2019 | Published: June 07, 2019

Keywords: Sinus; Sinus Pain; Sinus Pressure; Osteopathic Manipulative Treatment; Sinusitis

\section{Introduction}

Chronic sinusitis (CRS) also referred to as rhinosinusitis is a condition involving inflammatory disease of the nose and paranasal sinuses as defined by the Royal College of Surgeons guidelines (RCS) 2016 and cited in earlier research by Mendez-Sanchez et al. [1] and recent research Bergmark \& Pynnonen [2]. The etiology of chronic sinusitis is largely unknown or believed to be multifactorial; with inflammation, infection and obstruction of sinus ventilation suggested by RCS (2016). CRS is subcategorized as with and without polyps (Rosenfeld, 2015). CRS can cause two or more persistent symptoms (one of which must be nasal obstruction and/or nasal discharge and/or facial pain/pressure or loss of smell) that last for more than 12 weeks (NICE, 2018) unlike acute sinusitis which has an infective a etiology and commonly resolved within 12 weeks of onset. CRS affecting 1 in 10 UK adults cited the RCS (2016), is reported to have a significant impact on health-related quality of life, high health-care provision and significant days lost to industry.

Primary care for CRS often involves saline irrigation, nasal steroid spray, or both. The repeated use of antibiotics for CRS in primary is not recommended due to limited evidence of efficacy (RCS, 2016, p.6; UWS, 2017, p.47) Surgery for secondary care in UK (RCS) 2016 and similar in US cited in University of Western States (UWS) Clinical Standards, Protocols, and Education (CSPE) Care Pathway (2017). It appears that both the Primary clinical practice guidelines (CPGs) from the RCS (2016) and UWS CSPE Care Pathway (2017) recommend long-term use of nasal steroid spray for CRS. Bergmark \& Pynnonen [2] cited few studies have examined the uptake of this recommendation. However, Chong et al. [3] found there was an increased risk of epistaxis (high quality evidence).
Osteopathy is a system of diagnosis and treatment for an extensive range of medical conditions GOsC [8], but high-quality evidence research has been moderate for the efficacy of osteopathic manipulative technique (OMT), with most studies only pointing for relieving low back pain Licciardone et al. [13,14] More recent research on OMT to the head region is based on anatomical, physiological, neurological, vascular, muscular, articular and lymphatic systems Louveau et al. [16]. The rigorous studies of concept or efficacy for OMT have not been robust, as OMT has traditionally had an empirical basis rather than a research basis Ching [3].

The objective of this review focuses on the effectiveness of OMT in certain studies and articles which were assessed using a critical appraisal tool (CASP, 2018). As there is scarce evidence based systemic reviews and meta-analyses in OMT from randomized control trials (RCT's) to measure the efficacy of OMT on sinus pain and the beneficial outcomes in the United Kingdom, and so the search was expanded to include other manual therapies in European and United States.

Paul, Buser [19] identified the use of OMT (by Osteopathic physicians) in an emergency department for patients with low back pain, chest pain, torticollis, asthma and sinusitis demonstrated related symptoms could be ameliorated or eliminated with OMT. Orlandi et al. (2016) cited both CRS and asthma frequently co-exist in the same patient affected by similar triggers and co-factors, therefore treating either one of the conditions often has a beneficial effect on both. Raghavan and Jones (2000) found that there was 
little evidence to support the effect of complementary therapies (including osteopathy) over those of the placebo effect, cognitive dissonance and the natural resolution of many disease processes. Folweiler and Lynch (1995) demonstrated nasal specific technique as part of a chiropractic approach may be useful in treating CRS and sinus headache. Lee-Wong et al. [12] study of patients with CRS in outpatient allergy clinic, found OMT utilising direct pressure and sinus drainage technique improved sinus pain/congestions in patients. Jeffrey, et al. (2012) carried out a feasibility study of impact of integrative East-West medicine on Sino nasal symptoms and quality of life for patients with chronic sinusitis.

Mendez-Sanchez et al. [1] study suggested that manual therapy (including manipulation) treatment could be considered as an appropriate alternative treatment of chronic sinusitis. The University of Western States (UWS) Clinical Standards Care Pathway (2017) recommended 2 specific procedures out of 5 were upper cervical spine manipulation (joint dysfunction may be present as a viscera-somatic response to sinus irritation and Eustachian tube manipulation (Eustachian tube dysfunction often a comorbid condition of sinusitis), others were rapid dilation the nasal passages (not sinus balloon dilation); facial massage; lymph drainage and percussion recommended for management of CRS. The more recent observational case control study Petersen et al. [20] of neck pain and cervical musculoskeletal dysfunction, common among patients with self-reported sinus headaches maybe a contributing factor to headaches attributed sinusitis. This highlights a potential research to identify a wider population that could benefit from OMT for pain relief with less medication.

\section{Literature Review}

Lee-Wong et al. [12] an allopathic physician (studied OMT during her post-doctoral training) carried out a pilot study on 15 patients (who requested alternative therapies for CRS pain relief) to determine the efficacy of OMT as an adjunct to allopathic therapy to possibly minimize the use additional pain medications for relief of CRS discomfort symptoms. One OMT session only comprising of four direct pressure and 'milking' techniques and a sinus drainage technique (a total of 6 maneuvers) for nasal congestion carried out on patients as described in DiGiovanna \& Schiowitz [28] or a total of 18 minutes of treatment. The results reported decreased sinus pain/congestion after OMT.

\section{Limitations}

a. The outcome is not statistically significant $p=0.0012$ $(\mathrm{p}=<0.0001$ is significant)

b. Small sample size-15 patients.

c. No control groups included (sham treatment or no treatment for the control group). A suggestion of sham treatment or no treatment for the control groups can provide a new factor to the two groups (Denscombe, 2017). However, difficulty in proving osteopathy efficacy via RCT's due to the impossibility of double blinding and difficulties in conducting ineffective sham treatments Licciardone \& Russon [13].

d. Only a pain scale symptom score card was used for patients before and after OMT. A Sino nasal Outcome Test
Questionnaire (SNOT-22) a widely used, self-administered, quality of life questionnaire for patients with CRS could be included for understanding specific patient-reported outcomes. The history and severity of symptoms not included therefore data is difficult to compare in relation to normative data for chronic sinusitis patients.

e. There was no follow up with CRS patients to see how long the improvement of pain lasts.

\section{Strengths}

a. This study supports the perpetual argument in the context of more research into the effectiveness of OMT. An allopathic doctor who is interested in OMT and initiated this research for CRS patients with sinus pain. As suggested by King [11] an opportunistic study for encouraging interprofessional dialogue that will enhance the public acceptance of the services of all professions who use their hands in healthcare.

b. A relatively short trial just one session but effective in highlighting and advocating OMT that are commonly taught and used in osteopathic medicine.

c. Potential for further research on OMT that were integral part of osteopathic approach and principle in manual medicine Ward \& Greenman [31,34].

Mendez-Sanchez et al. [1] A prospective case series study in reporting manual therapy carried out on 14 patients diagnosed with CRS with craniofacial pain. The rational of the applied manual therapy is based on the premise that inadequate drainage preventing resolution of sinusitis; the autonomic nervous system is influenced by the imbalance of adrenergic, cholinergic, and sensory components; it seems that the parasympathetic stimulation increases nasal-mucosa secretions, whereas sympathetic stimulation causes circulatory vasoconstriction within the nose and sinuses. Hence the reasoning that manual therapy interventions targeting the thoracic and upper cervical segments with spinal manipulation as well as craniosacral treatments may influence the sympathetic and parasympathetic systems and ultimately have a positive effect on the autonomic nervous system. Hence integrating manual therapy with the overall management of symptoms associated with CRS may allow for reduced use of prescription medications.

The manual techniques include:

a. Thoracic spinal manipulation applied over T1-T3;

b. Manipulation of the cervicothoracic junction;

c. Manipulation of the pelvic girdle;

d. Decompression of the temporomandibular joint;

e. Myofascial release of the anterior cervical region;

f. Myofascial release of the hyoid system;

g. Mobilization of the maxilla bones;

h. Mobilization and compression of the frontal bone. 
The trials carried out for 7 weeks. Treatment carried out in Session 2, 3 and 5, evaluation only in session 1, 4, 6 and 7. Outcome measure shown a difference after treatment same for pain scores and pressure pain threshold (PPT). The results reported decreased sinus pain/congestion after OMT. $\mathrm{P}=<0.0015$.

\section{Limitations}

a. The outcome is not statistically significant $p=<0.0015$ ( $p=<0.0001$ is significant $)$

b. Small sample size-14 patients

c. No control groups included, cause and effect relationship cannot be determined

d. Complex outcome measures-3 questionnaires and pressure algometry testing at each session.

e. No indication of how long each treatment session took.

\section{Strengths}

a. Treatment was based on the presentation of the symptoms of the patient on each session.

b. A combination of local and regional manual therapy interventions appeared to yield improvements in all outcome measures.

c. Manual therapy interventions for patients appeared to fair better than when similar patients are treated with antibiotic drugs or endoscopic surgery.

d. Standardized evaluation methods with all sessions for each patient at the same period in the afternoon to reduce a confounding temporal effect

e. Potential for further research to look at medium- and long-term effects of manual therapy.
Jeffrey et al. (2012) carried out prospective, non RCTs to establish the feasibility of studying the impact of integrative EastWest medicine (IEWM) on Sino nasal symptoms and quality of life (QoL) on patients with CRS. Acupuncture, Acupressure, dietary modification and advice on stress management were introduced in addition to the standard medical therapeutic regimen.

\section{Limitations}
a. Not RCTs and absence of control groups.
b. Bias selection of study population.
c. Too many modalities of treatment were carried out.

d. Not an efficacy analysis but to obtain an estimate of the effect size and variance necessary to plan a definitive study to test and refine individual components of the IEWM protocol.

e. Small sample size-11 patients.

\section{Strengths}

a. First study using Integrated two health care systems Preliminary data indicates IEWM may improve symptoms and Quality of Life (QoL) for patients with CRS for future research

b. Over 8 weeks of regular reproducible treatment

c. Potential further research integrating OMT and Acupuncture

In summary, there is limited osteopathic research carried out with use of OMT to help reduce CRS and symptoms. The results showed deficiencies in the study design for Systemic Review and Meta-Analysis, the lack of control group in the RCTs. Nevertheless, the studies in this review have shown a clear justification the potential use of OMT along with traditional allopathic medicine, either locally Lee-Wong et al. [12] or locally and regionally MendezSSanchez et al. [1] to help easing sinus pain and congestion.

\begin{tabular}{|c|c|c|}
\hline \multicolumn{3}{|c|}{ Appendix 1A: 'frontal lift' OMT versus combined OMT. } \\
\hline OMT & Procedure & Time \\
\hline Streamlined 'frontal lift' to coronal suture & $\begin{array}{l}\text { - With the patient supine the operator stands at the head of the couch; } \\
\text { - With the head supported to avoid extension, the operator places the finger pads of } \\
\text { his index and mid fingers under the patients supra orbital ridge, care being taken not } \\
\text { to contact their eye } \\
\text { - Gentle pressure is then applied to lift the frontal bone from the nasal and ultimately } \\
\text { the ethmoid, which can be re-enforced by the operator } \\
\text { - gently griping the upper aspect of the nose with their thumb and index finger. There- } \\
\text { by opening the sinus ostium; } \\
\text { - The technique can be re-enforced by holding back on the nasal bone; } \\
\text { Re-assess. } \\
\text { Lintonbon (2013) }\end{array}$ & 3 mins \\
\hline
\end{tabular}




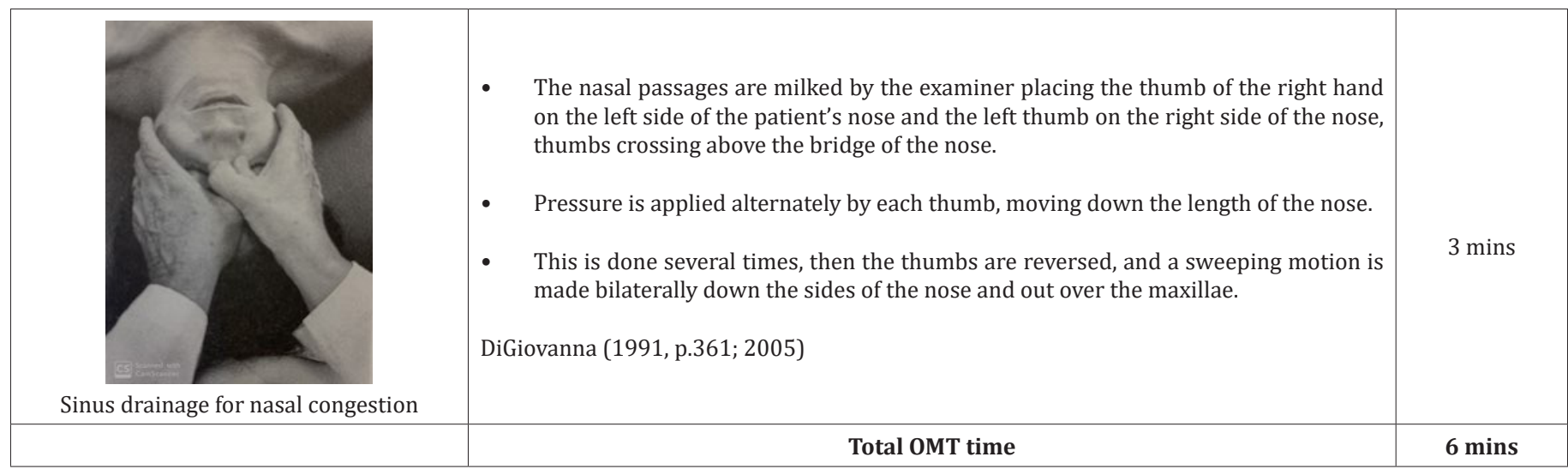

Lee-Wong et al. [12] offers a viable opportunity for further research. Building on from this study by investing the effect of 'frontal lift' OMT - a craniosacral technique to release restricted side of the frontal bone to achieve balance of both sides to affect sinus congestion in the ethmoid or frontal sinuses cited in DiGiovanna \& Schiowitz [28,29] and Greenman [31], the potential benefits of using a streamlined technique requiring less treatment time, may improve patients' comfort. Therefore, affording the practitioner more time available to treat the patient's other tissues causing symptoms. This streamlined 'frontal lift' OMT to coronal suture claims to open the coronal suture and therefore opens the drainage passages of all sinuses as shown by Lintonbon [15]-See Appendix $1 \mathrm{~A}$-to ease facial-maxillary sinus headache. Hence this study is to test the streamlined 'frontal lift' OMT use on its own is sufficed, comparing with the combined OMT (Appendix 1B) for CRS patients. Therefore, a shorter treatment time by $60 \%$ (reduced from 18 mins to 6 mins). Thereby enhancing patient comfort (Appendix 1A).

\section{Aim of the Quantitative study}

The main aim of this study is to investigate the effect of 'frontal lift' OMT compared with combined OMT (direct pressure and "milking" techniques and sinus drainage technique) in easing CRS and congestion.

\section{Objectives of Quantitative study}

From the studies reviewed, a sample size with minimum 16 participants have been chosen. 2 experimental groups, 1 control group. Sample size of 48 . a. To collect data obtained using a Sino nasal Outcome Test Questionnaire (SNOT-22)

b. To use a Numeric Pain Rating Scale (NPRS) experienced by the participants, before and after the application of the 'frontal lift' technique on its own compared with pain experienced by participants before and after the application of the 'combined techniques' (direct pressure and "milking" techniques and one sinus drainage technique)

c. From the data collected from the 2 experimental groups and a control group, it could be determined which is the more effective technique (s) to reduce symptoms of pain and congestion with chronic sinusitis

d. To select participants and randomly allocate them into 2 different groups, Group A\&B as well as a Control Group

e. To apply 'frontal lift' technique to Group A; to apply 'combined techniques (direct pressure and "milking" techniques and sinus drainage technique) to Group B and Control Group with sham treatment or no treatment

f. To compare SNOT-22 questionnaire pre and post treatment and NVPR scores between Group A, Group B and Control Group

g. To analyses the data obtained from the SNOT-22 from the 3 groups

h. To determine if the 'frontal lift' OMT on its own is just as effective as using a combined OMT (direct pressure and "milking" techniques and a sinus drainage technique) in easing sinus pain and congestion (Appendix 1B).

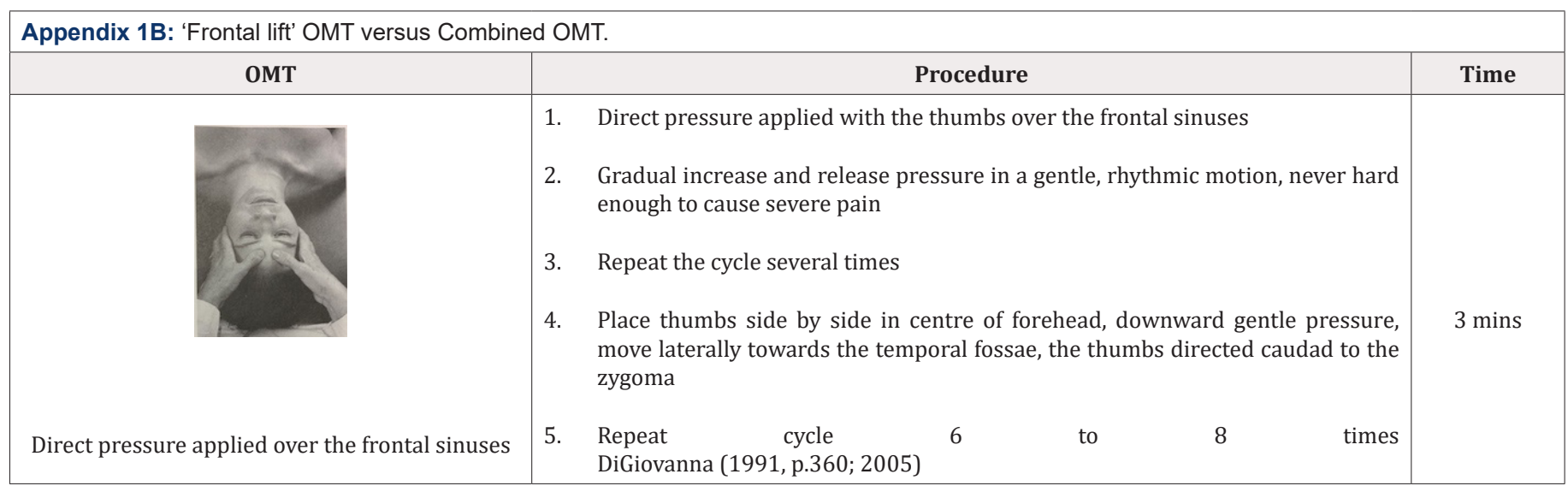




\begin{tabular}{|c|c|c|}
\hline $\begin{array}{l}\text { Gentle pressure applied over the supraorbital } \\
\text { notch }\end{array}$ & $\begin{array}{l}\text { 1. Gentle pressure applied over the supraorbital } \\
\text { 2. Then the thumbs swept along the eyebrow ridge bilaterally } \\
\text { DiGiovanna (1991, p.360; 2005) }\end{array}$ & 3 mins \\
\hline 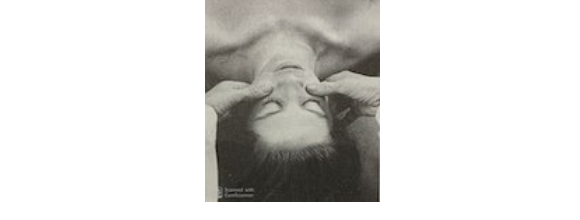 & $\begin{array}{l}\text { 1. Pressure applied over the sinuses with the thumbs } \\
\text { 2. The nasal passages are "milked" by beginning with the thumb on each side of the } \\
\text { nose and pressing down while sweeping the thumbs laterally along the maxilla } \\
\text { DiGiovanna (1991, p.360; 2005) }\end{array}$ & 3 mins \\
\hline Direct proccure & $\begin{array}{l}\text { 1. Pressure applied over the temporal areas by gently placing the thenar eminenc- } \\
\text { es in the temporal fossae bilaterally } \\
\text { 2. Compressing these areas between the hands } \\
\text { 3. Pressure is applied and released in gentle, rhythmic motions } \\
\text { DiGiovanna (1991, p.360; 2005) }\end{array}$ & 3 mins \\
\hline Sinus drainage for nasal congestion & $\begin{array}{l}\text { 1. The nasal passages are milked by the examiner placing the thumb of the right } \\
\text { hand on the left side of the patient's nose and the left thumb on the right side of } \\
\text { the nose, thumbs crossing above the bridge of the nose. } \\
\text { 2. Pressure is applied alternately by each thumb, moving down the length of the } \\
\text { nose. T } \\
\text { 3. This is done several times, then the thumbs are reversed, and a sweeping motion } \\
\text { is made bilaterally down the sides of the nose and out over the maxillae. } \\
\text { DiGiovanna (1991, p.361;2005) }\end{array}$ & 3 mins \\
\hline & Total OMT Time & $18 \mathrm{mins}$ \\
\hline
\end{tabular}

\section{Experimental hypothesis}

Using a 'frontal lift' OMT is just as effective at reducing the painful symptoms in chronic sinusitis patients at the LSO Clinic, London, in comparison to patients receiving a combined OMT.

\section{Null hypothesis}

Using a 'frontal lift' OMT is less effective at reducing the painful symptoms in CRS patients at the LSO Clinic, London, in comparison to patients receiving a combined OMT.

\section{Approach \& Methodology}

The research strategy adopted in this study is a quantitative research approach utilizing a Randomized Controlled Trials (RCTs) experiment design to test the hypothesis. Randomized study design reduces bias and a rigorous tool to examine causeeffect relationships between an intervention and outcome
Hariton \& Locascio [9]. Other advantages include repeatability of identical procedures, precision and consistency in gathering of data. However, Denscombe [27] also cited disadvantages such as artificial settings, ethics in this instance, the use of control groups with sham or no treatment, therefore control of the relevant variables maybe difficult to achieve. The difficulties in constructing a sham technique, if all touch especially the face can induce reward or placebo - induced pain control, then differentiating the effects of OMT and a sham technique can prove to be tricky (Petrovic, et al., 2002; Zubieta, et al., 2005).

\section{Methods-Participants}

My sample size of 48 participants will be determined through LSO Clinic (3 groups of 16 participants in each group). There will be 2 groups of CRS participants and a control group receiving no treatment or sham treatment (Table 1). 
Table 1: Eligibility Criteria of Participants for Chronic sinusitis.

\begin{tabular}{|c|c|}
\hline Inclusion Criteria & Exclusion Criteria \\
\hline $\begin{array}{l}\text { - Adult males and females aged over } 18 \\
\text { - } \\
\text { - } \text { Any ethnic background, sexual orientation and religious beliefs } \\
\text { more of the following signs and symptoms: } \\
\text { o mucopurulent drainage (anterior, posterior, or both) } \\
\text { o nasal obstruction (congestion) } \\
\text { o facial pain-pressure-fullness, or } \\
\text { - Commitment for participant availability in the study } \\
\text { - Strong understanding of the English language }\end{array}$ & $\begin{array}{l}\text { - Minimum age over } 18 \\
\text { - Had contraindications for manual therapy, including spinal ma- } \\
\text { nipulation } \\
\text { - } \quad \text { Had history of cranial, facial or neck surgery } \\
\text { - } \quad \text { Had complications associated with CRS } \\
\text { during prescription medications for their presenting symptoms } \\
\text { - Had received manual therapy interventions for their condition } \\
\text { within } 2 \text { months before the study } \\
\text { Refrain from starting any new therapeutic interventions or med- } \\
\text { ications during their participation } \\
\text { - Have any co-existing conditions that may impact on their ability } \\
\text { to interact in conversation } \\
\text { Limited mental capacity to give informed consent through verbal } \\
\text { and written information } \\
\text { Current critical stage of their condition making them unable for } \\
\text { participation }\end{array}$ \\
\hline
\end{tabular}

\section{Methods-Study Design}

Building on from Lee-Wong pilot study a more 'robust' study design to include:

1. Participants in the study will be seen once a week for 6 weeks.

2. The use of SNOT-22 before treatment at each week. (Appendix 2)

3. The use of NPRS before and after end of treatment from the 1 st week to 6 th week to observe an improvement in easing sinus pain and congestion. (Appendix 3). The Numeric Pain Rating Scale (NPRS) has good sensitivity and generates data for analysis Karcioglu et al. [10].

4. Bigger sample size-48

a. Streamlined frontal lift' OMT (Group A)-16 peopletreatment time: 6 mins each

b. Combined OMT (Group B)-16 people-treatment time: 18 mins each

c. Control group (Group C-sham or no treatment)-16 people

\section{Methods-Outcome Measures}

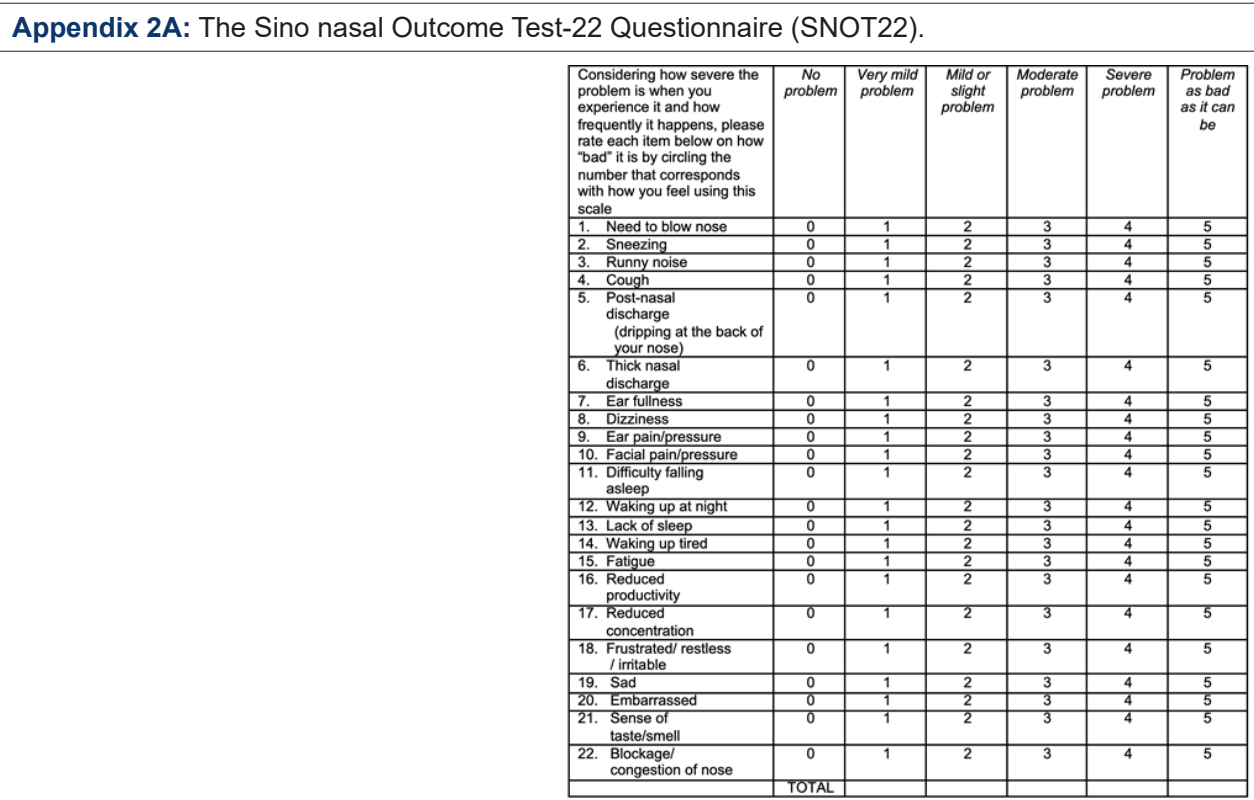


In order to select 48 patients clinically diagnosed with CRS, a Sino nasal Outcome Test Questionnaire (SNOT-22)- Appendix 2-is chosen based on the highest quality for understanding specific patient-reported outcomes during the management (PROM) of chronic sinusitis (CRS) showed in a recent systemic review Rudmik et al. [22] (Table 2 \& Appendix 2a).

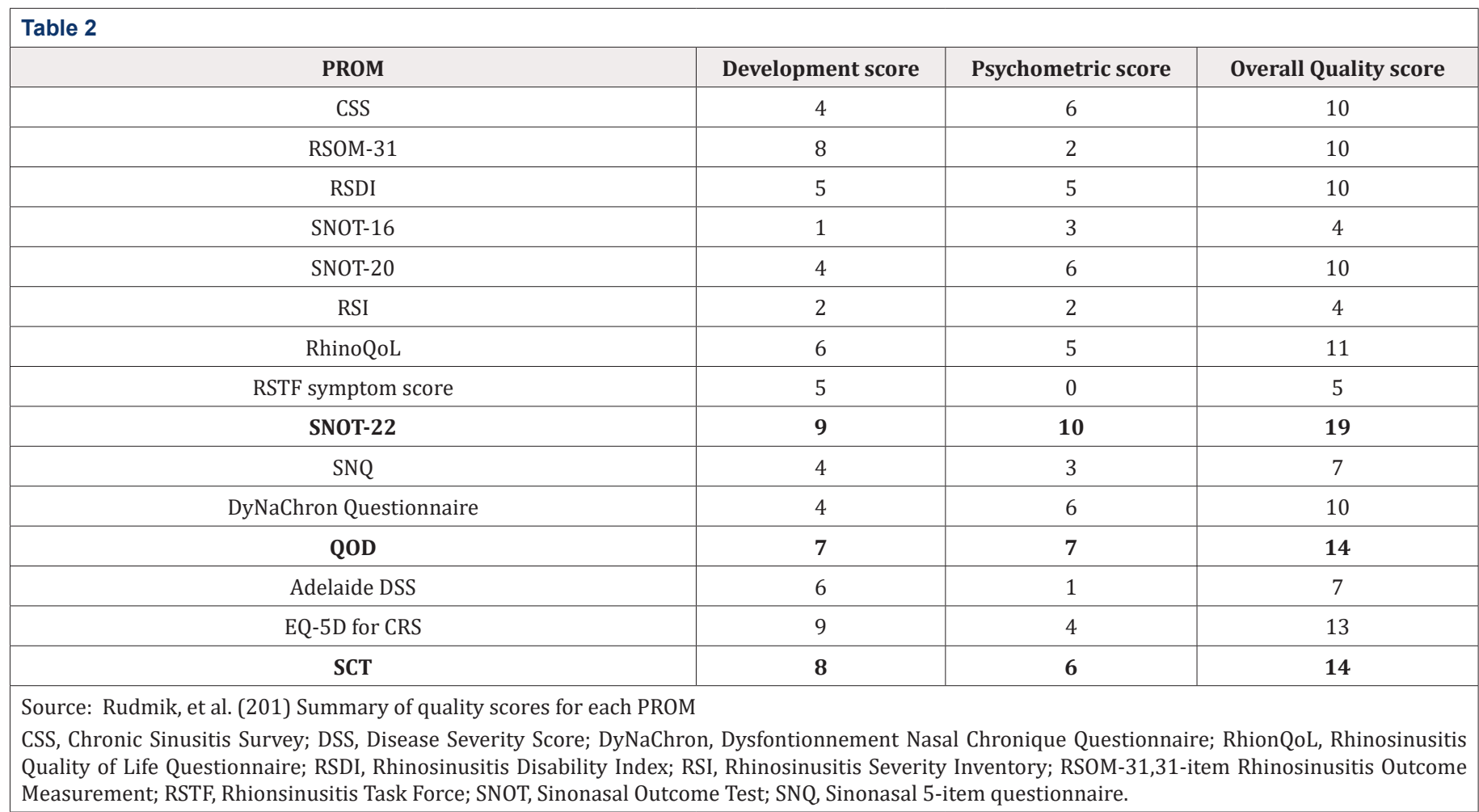

The independent variable includes 'frontal lift' OMT and combined OMT which allow the researcher to consider if either one of the two OMT techniques leads to the dependent variable in reduction of pain symptoms. Most importantly, the researcher must investigate whether the independent variable of OMT influences the dependent variable to agree with its experimental hypothesis.

\section{Data Analysis}

The data from the SNOT-22 and NPRS will be analysed using SPSS Version 24 software, because of its extensive statistical data analysis capability, as well as effective data management within an excel format (Stehlik-Barry and Babinec, 2017). It will be accessed and interpreted as ordinal data through extensive statistical analysis by using the SPSS software. Using the nomogram (Field, 2017) data will be analysed along this type of format: Ordinal data ? Differences between groups ? Dependent ? ordinal rank ? Non-parametric (rank test Wilcoxon matched pairs signed) ? NonParametric (Friedman's 2-way analysis of variance by ranks).

The sum test (Wilcoxon rank) will check the frequency of conditions between the two groups e.g. amount of pain reduction. The Friedman's test will compare the division of the 2 quantitative variables, with analysis of variance (ANOVA) for dependent samples (Flow chart).

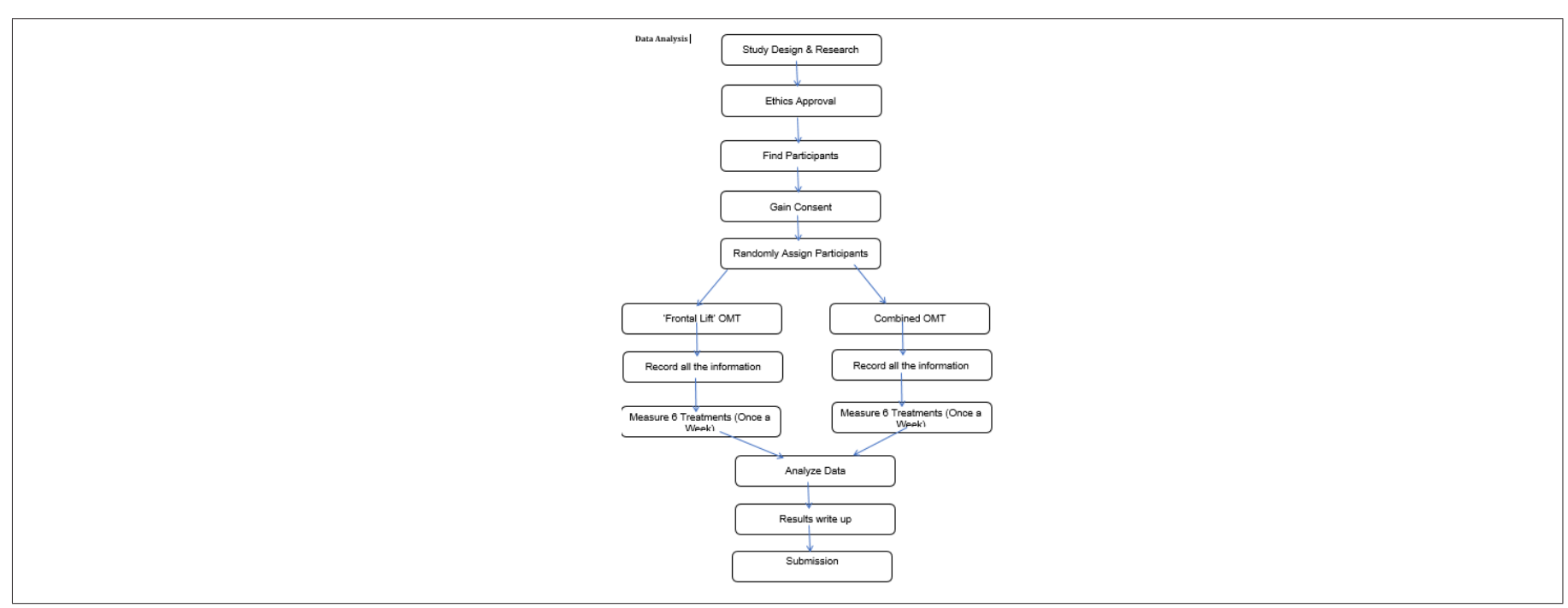




\section{Resources Required}

a. The Sino nasal Assessment Questionnaire (SNOT-22)

b. Numeric Pain Rating Scale (NPRS)

c. SPSS Version 24 Software-download from ARU

d. Access to room at LSO clinic for treatment process e. Poster advertisements (including any exclusion criteria) of the study placed in LSO's reception waiting area to invite volunteers to participate in the study.

f. Letter and Consent form (see ethical issues-checklist no.2) (Appendix 2b).

Appendix 2B: Numeric Pain Rating Scale (NPRS).

$\left.\left.\left.\left.\left.\left.L_{\text {no pain }}^{0} \perp^{1}\right|^{2}\right|^{3}\right|_{\text {moderate pain }} ^{4}\right|^{5}\right|^{7}\right|_{\text {worst possible pain }} ^{8}$

The Numerical Pain Score Rating Scale (NRS)

\section{Literature Search Strategy}

An extensive literature search was conducted using several search databases with access gained via Anglia Ruskin University Library, using MEDLINE, PubMed, Google scholar, Science Direct, Cochrane database/library, and Europe PMC. The keywords used (in various combinations) to search the literature were: osteopathic, medicine, osteopathy, Rhinosinusitis, sinusitis, sinus, adult sinusitis, headache, asthma, pain, craniofacial pain, pressure, neck, manipulation, chronic, manipulative, treatment, effects, manual, therapy, techniques, allergy, chiropractic, quality of life, review, outcome, measures, alternative, complementary, randomized controlled trials. Boolean operators (AND and OR) were used e.g. Osteopath\# AND Sinus\#.

Also, London School of Osteopathy (LSO) library resources were used to access hard copy materials such as books and journals for additional evidence (Table $3 \& 4$ ). The database search dates were conducted from March 9th -1st May 2019 for further citations of this research.

\begin{tabular}{|c|c|}
\hline Inclusion Criteria & Exclusion Criteria \\
\hline $\begin{array}{l}\text { - Published Systemic reviews, primary research, experimental } \\
\text { study, articles, case studies, books, commentaries } \\
\text { - } \\
\text { - } \\
\text { English studies, clinically relevant to this topic. } \\
\text { and still relevant } \\
\text { - Emphasis on literature from within the UK and studies from oth- } \\
\text { er geographical regions. }\end{array}$ & $\begin{array}{l}\text { - Children Sinusitis } \\
\text { - Non-English publications/studies of different physical therapies } \\
\text { irrelevant to this research. } \\
\text { - Sources focused on osteopathic treatment towards other patho- } \\
\text { logical conditions aside from Sinusitis would be inconsistent } \\
\text { with the research data. } \\
\text { - Exclusion of unpublished osteopathic studies. } \\
\text { - Articles that was purely theoretical. }\end{array}$ \\
\hline
\end{tabular}

\begin{tabular}{|c|c|}
\hline Table 4: Literature search strategy. & Number of papers \\
\hline Type of research & 6 \\
\hline Systematic Reviews/Meta-Analysis & 3 \\
\hline Randomized Control Trials & 1 \\
\hline Questionnaires/Survey & 4 \\
\hline Case Control Studies & 2 \\
\hline Case Studies & 2 \\
\hline Narrative Review & 5 \\
\hline Books & $\mathbf{2 3}$ \\
\hline TOTAL & \\
\hline
\end{tabular}

\section{Osteopathic Relevance}

DiGiovanna et al. [28] found that chronic sinusitis is almost always associated with somatic dysfunction of the cervical spine. Sympathetic innervation to the sinuses arises from the upper thorax and travels through the cervical region. Hence treating cervical somatic dysfunction and performing sinus drainage techniques helps to relieve pain and as well as assist in the drainage of the sinuses. These techniques used are fully explained in most osteopathic handbooks. However, none of them fully explain the effectiveness of one technique over another. This study is to investigate a shorter treatment with one technique as opposed to using several. This would deliver maximum pain relief from OMT 
and therefore improve the patients' comfort leading to improved treatment outcome in a shorter time scale allowing the practitioner to work on associated tissues causing symptoms.

From a quantitative perspective, the study design will contribute towards creating additional research towards the field of osteopathy by assessing if a streamlined OMT is just as effective compared with a combined OMT technique therefore potentially to improve patients' comfort with less treatment time. This could support the relevance of osteopathy in establishing whether this manual technique 'frontal lift' can be a significant factor contributing towards an individual's health. Osteopathy is widely used as a therapeutic tool; however, its reliability as a form of treatment remains unconfirmed. Therefore, research regarding chronic sinusitis treatment using OMT should be conducted to highlight future research priorities.

\section{Limitations}

Despite the best intentions and following the guidelines, the lack of recent research in the osteopathic field. Hence the generation of a hypothesis from the data collected is rather subjective. Also, the use of a placebo, control group and sham treatments have been overlooked/not included by the researchers in the reviewed articles.

\section{Conclusion}

The use of 'frontal lift' OMT appears to be less invasive and suitable for a wide range of CRS patients. The shorter the time of treatment allowing the practitioner to work on the potential maintaining factors thereby working on the body as a whole - one of the core principles of osteopathy. The extra time generated also allows the practitioners to explore the patient's biopsychosocial aspects of the patient.

\section{Ethical Issues -Checklist (Table 5).}

\begin{tabular}{|c|c|}
\hline \multicolumn{2}{|c|}{ Table $\mathbf{5}$} \\
\hline All ethics must be approved by the ethics committee and faculty of health sciences in Anglia Ruskin University (ARU) (Scott, 2016). \\
\hline 1. & $\begin{array}{c}\text { Participants are invited for voluntary participation when attending the LSO clinic. This is handled through LSO clinic office to avoid coercion. } \\
\text { about the research and risks, e.g. possible side effect after treatment; state commitment required for the number of treatments; state right for } \\
\text { withdrawal from participation at any time without penalty and obtain informed consent from participants. Adequate timing for participants to } \\
\text { decide and discuss this with family and friends first before proceeding. }\end{array}$ \\
\hline 3. & All fairness and equality is ensured for all participants. \\
\hline 4. & Anonymity to be assured by allocating a number instead of a using the patient's name. \\
\hline 5. & $\begin{array}{r}\text { Patients understand that participation is voluntary, have the right to withdraw at any time without affecting patient's future treatment experi- } \\
\text { ences. }\end{array}$ \\
\hline 6. & $\begin{array}{r}\text { Health and Safety Risk Assessment completed to ensure that patients' comfort and that they do not undergo psychological trauma as a result of } \\
\text { the treatment. }\end{array}$ \\
\hline 7. & Any complaints from the study should be handled through ARU procedures. \\
\hline 8. & $\begin{array}{c}\text { All data collected needs to comply with latest GDPR. The information must be kept on password-protected computer. Collected data will be held } \\
\text { securely according to the Data Protection Act 1988. The confidentiality of participants is assured as collected information will be anonymised } \\
\text { and the data is only available to the researcher. The data will be destroyed after the study has ended. }\end{array}$ \\
\hline 9. & The study will be covered by Anglia Ruskin University insurance once ethical approval is obtained. \\
\hline 10. & All participants are entitled to their feedback results via email or post. \\
\hline
\end{tabular}

\section{References}

1. Bergamini MVM, Licciardone JC, Kearns CM, Hodge LM (2012) Associations of cytokine concentrations with key osteopathic lesions and clinical outcomes in patients with non-specific chronic low back pain: results from the osteopathic trial. Journal of the American Osteopathic Association J Am Osteopath Assoc 112(9): 596-605.

2. Bergmark RW, Pynnonen M (2017) Diagnosis and first-line treatment of chronic sinusitis. JAMA 318(23): 2344-2345.

3. Ching LM (2016) Research into osteopathic manipulative medicine: steps on the evidence pyramid. J Am Osteopath Assoc 116(3): 133-134.

4. Critical Appraisal Skills Programme (2018) CASP (Systematic Review) Checklist.

5. Critical Appraisal Skills Programme (2018) CASP (Randomized Controlled Trial) Checklist.

6. Critical Appraisal Skills Programme (2018) CASP (Diagnostic Test Study) Checklist.

7. Critical Appraisal Skills Programme (2018) CASP (Clinical Prediction Rule) Checklist.
8. General Osteopathic Council (2019) What Osteopathy is?

9. Hariton E, Locascio JJ (2018) Randomised controlled trials - the gold standard for effectiveness of research. BJOG 125(13): 1716.

10. Karacioglu O, Topacoglu H, Dikme O, Dikme O (2018) A systematic review of the pain scales in adults: Which to use? Am J Emerg Med 36(4): 714-715.

11. King HH (2011) Osteopathic manipulation may be beneficial in the treatment of patients with chronic sinusitis: review article - the somatic connection. Journal of the American Osteopathic Association 111(10): 569-570.

12. Lee-Wong M, Karagic M, Doshi A, Gomez S, Resnick D (2011) An osteopathic approach to chronic sinusitis. Journal of Allergy \& Therapy $2(2)$.

13. Licciardone JC, Brimhall AK, King LN (2005) Osteopathic manipulative treatment for low back pain: a systemic review and meta-analysis of randomized controlled trials. BMC Musculoskelet Disord 4(6): 43.

14. Licciardone JC, Franke H, Franke JD, Fryer G (2014) Osteopathic manipulative treatment for nonspecific low back pain: a systematic review and meta-analysis. BMC Musculoskelet Disord 30(15): 286. 
15. Lintonbon D (2013) Osteopathic approach to the treatment of facialmaxillary sinus headache. Positive Health Online Pp. 206.

16. Louveau A, Smirnov I, Keyes TJ, Eccles JD, Rouhani SJ (2015) Structural and functional features of central nervous system lymphatic vessels. Nature 523(7560): 337-341.

17. Méndez-Sánchez R, Gonzalez-Iglesias J, Puente-Gonzalez AS, SanchezSanchez JL, Puentedura EJ, et al. (2012) Effects of manual therapy on craniofacial pain in patients with chronic rhinosinusitis: A case series. Journal of Manipulative \& Physiological Therapeutics 35(1): 64-72.

18. Ngyyen DT, Rumeau C, Felix-Ravelo M, Nguyen-Thi PL, Jankowski R (2017) Sino nasal symptom assessment by the self-reported Dy Nachron questionnaire: Before or after consultation? European Annals of Otorhinolaryngology, Head and Neck diseases 134(1): 19-22.

19. Paul FA, Buser BR (1996) Osteopathic manipulative treatment applications for the emergency department patient. The Journal of the American Osteopathic Association 96(7): 403-409.

20. Petersen SM, Jull GA, Learman KE (2019) Self-reported sinus headaches are associated with neck pain and cervical musculoskeletal dysfunction: a preliminary observational case control study. J Man Manip Ther 4: 1-8.

21. Raghavan U, Jones NS (2000) Complementary and alternative therapy for nasal conditions: review article. The Journal of Laryngology \& Otology 114: 919-924.

22. Rudmik L, Hopkins C, Peters A, Smith TL, Schlosser RJ, et al. (2015) Patient-reported outcome measures for adult chronic rhinosinusitis: A systematic review and quality assessment. J Allergy Clin Immunol 136(6): 1532-1540.
23. Scott J (2017) Code of practice for applying for ethical approval at Anglia Ruskin University. Version 7.2

24. Royal College of Surgeons (2016) Commissioning guide: Chronic Rhinosinusitis.

25. Health Centers of University of Western States (2017) Clinical standards, protocols and education (CSPE) care pathway. Sinus pain and rhinosinusitis.

26. Cottrell S (2011) Critical thinking skill. $2^{\text {nd }}$ Edn Plagrave Macmillan Ltd, New York.

27. Denscombe M (2017) The good research guide for small research project. $6^{\text {th }}$ Edn Open University Press, Maidenhead, England.

28. DiGiovana EL, Schiowitz S (1991) An osteopathic approach to diagnosis and treatment. Lippincott, USA.

29. DiGiovana EL (2005) An osteopathic approach to diagnosis and treatment. Lippincott, USA.

30. Field A (2017) Discovering statistics using SPSS (Introducing statistical methods series). $5^{\text {th }}$ Edn Sage Publication Ltd, London.

31. Greenman PE (2003) Principles of manual medicine. $3^{\text {rd }}$ Edn Lipppincott, USA.

32. Stehlik-Barry K, Babinec AJ (2017) Data analysis with IBM SPSS statistics.

33. Birmingham, Packt Publishing.

34. Ward RC (1997) Foundations for osteopathic medicine: American Osteopathic Association. LW\&W, USA 